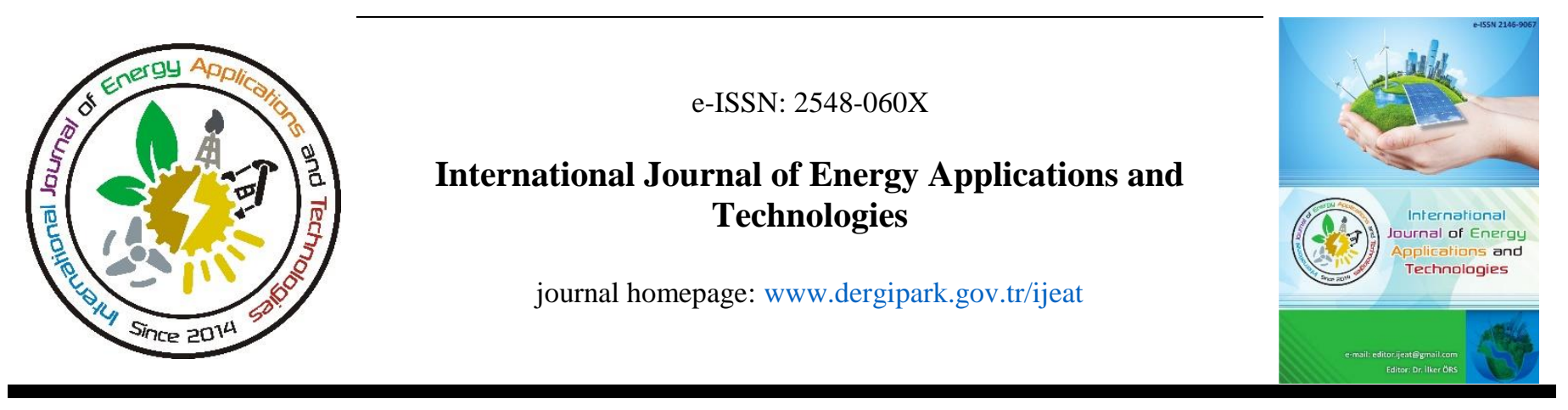

Original Research Article

\title{
Leakage detection in underwater oil and natural gas pipelines using convolutional neural networks
}

\author{
Ayşegül Avcı, Seda Karadeniz Kartal ${ }^{*}$ \\ Department of Electrical and Electronics Engineering, Zonguldak Bülent Ecevit University Zonguldak, Türkiye
}

\author{
ARTICLE INFO \\ * Corresponding author \\ sedakartal@beun.edu.tr \\ Received October 1, 2020 \\ Accepted December 20, 2021 \\ Published by Editorial Board \\ Members of IJEAT \\ (C) This article is distributed by \\ Turk Journal Park System under \\ the CC 4.0 terms and conditions.
}

doi: $10.31593 /$ ijeat. 803960

\begin{abstract}
Underwater oil and natural gas pipelines are an underwater transport infrastructure known to be reliable, fast, and efficient, preferred for the transmission of energy to far distances. The rapid and continuous increase in demand for energy due to population growth, industrial developments, and global growth requires economic and environmental solutions for the safe transmission and control of energy sources such as oil and natural gas. These lines are damaged due to their work in corrosive ambient conditions, natural elements such as sudden change of air and water temperatures, tectonic activities, and external elements such as blows caused by fishing equipment and military exercises. Therefore, it is necessary to determine the damages without requiring more hardware, saving time, and cost. In this study, underwater oil and natural gas pipelines were detected using convolutional neural networks and the detection performance of artificial neural network was analyzed. Underwater pipelines are detected using convolutional neural networks with $97.63 \%$ accuracy. A reliable, fast, efficient, controlled, and sustainable model is established to prevent potential damage to underwater pipelines from becoming an environmental threat to water and air pollution and living creatures in the underwater ecosystem with this study.
\end{abstract}

Keywords: Convolutional neural networks; Underwater object detection; Underwater oil and natural gas pipelines; Underwater pipelines detection; Unmanned underwater vehicle

\section{Introduction}

Today, underwater research is conducted in civilian and military applications for different and varied purposes, such as protection and investigation of natural and environmental resources, various construction activities, ensuring coastal and country security. Particularly over the past two decades, a major part of the academic and industrial research has focused on the use of unmanned platforms in order not to put human lives at risk. The use of unmanned underwater vehicles is becoming increasingly widespread in areas such as archaeology and submerged studies, minesweeping, recognition and destruction, bridge pier controls, underwater observation and exploration, underwater mapping, oceanographic research, and fracture/ crack/ leakage detection on underwater oil and natural gas pipelines [1].
Underwater pipelines are submerged lines that are used for the transmission of liquids and gases to long distances. Underwater transportation is preferred and recognized as one of the most reliable, fastest and most efficient ways to transmit energy from production centers to demand centers [2]. Natural gas and oil pipelines are used for the transmission of energy sources of strategic importance in Turkey and the world. About $70 \%$ of the world's energy transmission lines are carried by pipelines. In addition to this, a wide range of hazardous liquids such as LPG, anhydrate ammonia, crude oil, gasoline and other refined petroleum products are also transported by pipelines [3]. Factors such as population growth and global growth increase the requirement and demand for these energy sources. According to the International Energy Agency (IEA) World Energy Outlook 
2019 Report, oil demand is projected to increase by $50 \%$ and natural gas demand by $40 \%$ until 2040 . This requires a sustainable system that is reliable and harmless to nature for underwater life and underwater pipelines.

In the material requirements (MR 0175) standard published by the National Association of Corrosion Engineers (NACE), it defines fluids containing water as liquid and hydrogen sulfide $\left(\mathrm{H}_{2} \mathrm{~S}\right)$ with a partial pressure greater than 0.0035 bar as the acidic gas atmosphere. They contain an acidic corrosive environment due to $\mathrm{H}_{2} \mathrm{~S}$, where they contain fluid liquids such as natural gas and oil transported in pipelines. For this reason, natural gas and oil pipelines also operate in corrosive environments [4]. Corrosion- type cracks caused by hydrogen sulfide, ground properties, weather and sudden changes of water temperature, thermal expansion, and contraction, subsidence, tectonic movements, waves and flows, salinity, seawater resistivity, $\mathrm{pH}$ value, wind, rain, ice formation, hydrostatic loads, military exercises, the blows of fishing equipment, mechanical accidents cause leaks in oil and natural gas pipelines [2], [5]. For these reasons, operational supervision, control, and monitoring are needed for the safe working conditions for pipelines.

\section{Convolutional Neural Networks}

Convolutional neural networks are a type of deep learning architecture with a multi-layered detector model that combines the convolution process and artificial neural networks. Deep learning is a sub-branch of machine learning where increasingly more useful representations can be obtained while processing data in successive layers [6]. Deep learning is a method based on learning from the representation of data.

Representation for an image can be thought of as a vector of density values per pixel, or as properties of edge clusters, such as custom shapes. Some features represent data better [7]. On the input given in the first layers, feature extraction is implemented through filters. At the same time, dimensioning functions are used to reduce the cost of computation and to transfer the summary information of the features learned from the input to other layers. Then these properties obtained from the input are converted into a onedimensional vector and given as input to the fully connected layer or layers and the classification process is implemented [8]. The following figure (Fig. 1) shows the structure of the convolutional neural network. The image input layer at the left of the image, followed by the convolution layer, the blue colored circles at the right of the image represent the output layer of the neural network, and the blue-colored circles at the right of the image represent the hidden layers.

The purpose of the convolution process is the feature extraction from the data. In the convolution process, layers learn regional patterns. The network learns small regional patterns with the first convolution layer, while the subsequent convolution layers learn wider patterns.

The convolution process is based on the fact that a small-size matrix taken over the image is shifted over a certain step length and the multiplication of each matrix with which it is shifted, the multiplication results are added and placed in the output matrix. The small-size matrix taken over the image is called the convolution filter and the output matrix is called the feature map. Each feature map creates the input of the subsequent layer. On images, areas such as edges, sharp-soft, and light-dark have an important place in feature mapping. Edges are calculated in the first layers of the convolutional model through edge-finding filters.

Data input and output sizes can be of different sizes due to the edge effect and the convolution process. Padding is done by adding pixels to the input matrix to equalize the input and output sizes.

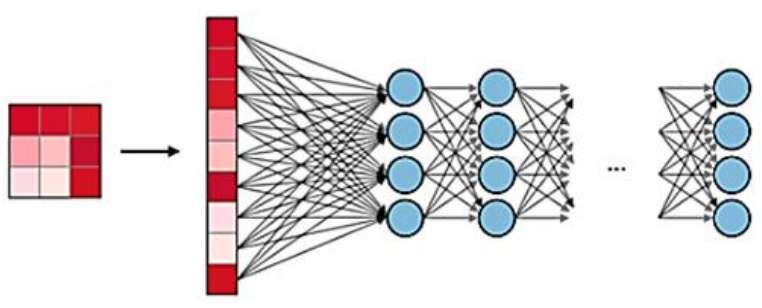

Fig.1. Convolutional neural network [1]

Then, in order to reduce the size of the feature map, pooling (the size reduction process) is performed. This process causes feature loss but reduces the training time of the network.

In the pooling process, small-sized matrices are extracted from the feature map, similar to the convolution process. A feature map is then re-created by taking the maximum value of each color channel. The layer operations in the convolutional neural network shown in Fig. 2.

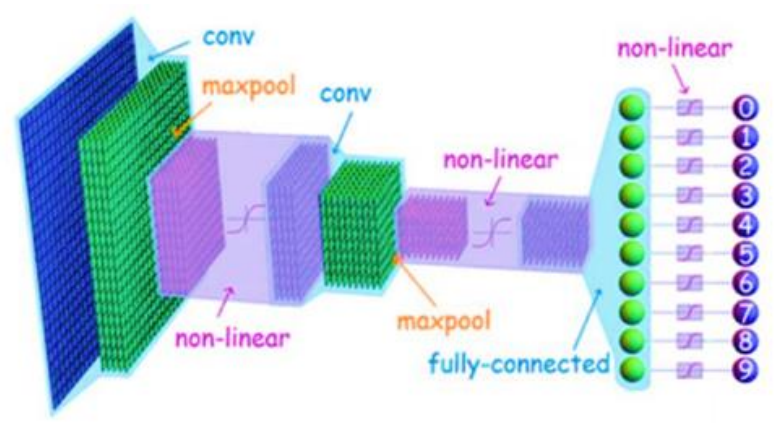

Fig.2. Operations in convolutional neural network [2]

\section{Underwater Pipeline Detection}

Low contrast ratio and turbidity occur on underwater images due to environmental effects such as diffraction properties of light in water, ambient conditions, and the effect of imaging subsystems such as pixel deviations. 
During the pre-processing of the data, images are clarified using filtering and contrast propagation methods. After the data is received and the initial model and algorithm evaluations are made, the data augmentation and preprocessing operations are applied to the existing data. The main reason for applying these operations is that the dataset represents the problem in a good way and to increase the performance rate of the model created for solving the problem. Synthetic data is acquired, and the number of data is increased by using rotation, scaling, cropping, and decolorizing methods on the data. Fig. 3 is shown the original image, Fig. 4, Fig. 5 and Fig. 6 are shown examples of data augmentation.
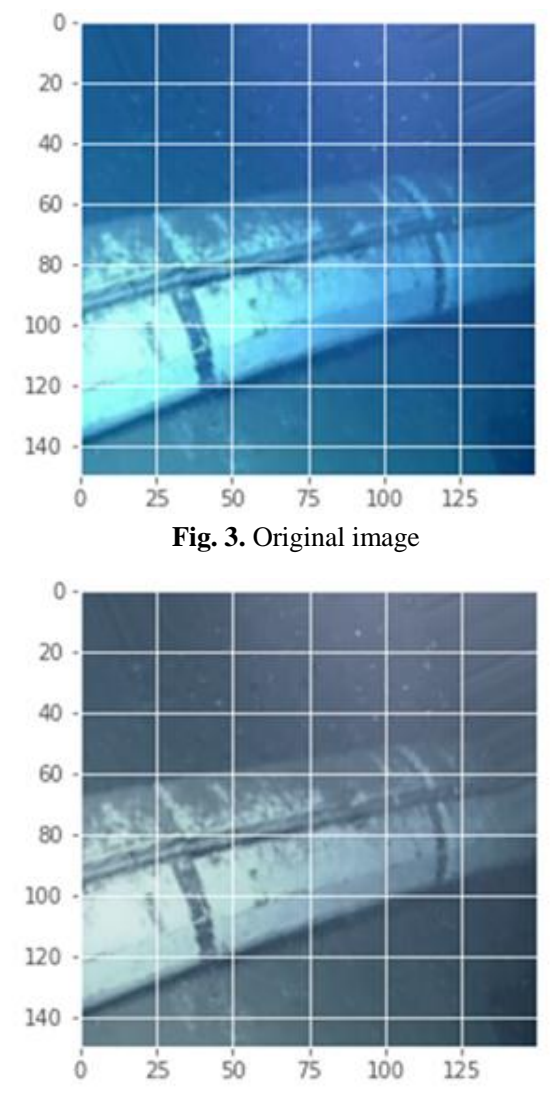

Fig. 4. Example of data augmentation

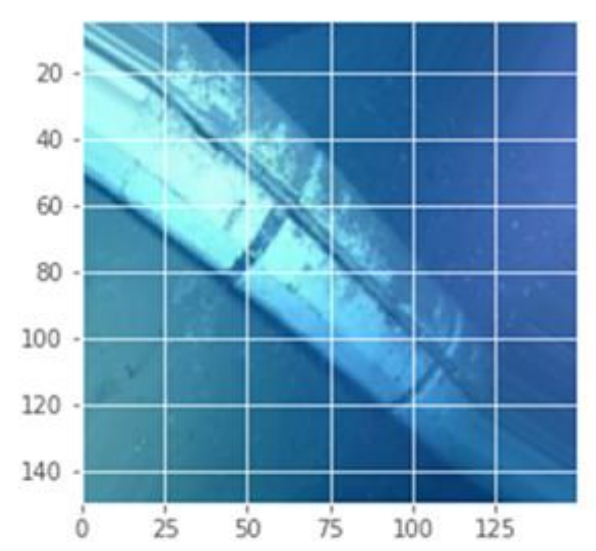

Fig. 5. Example of data augmentation

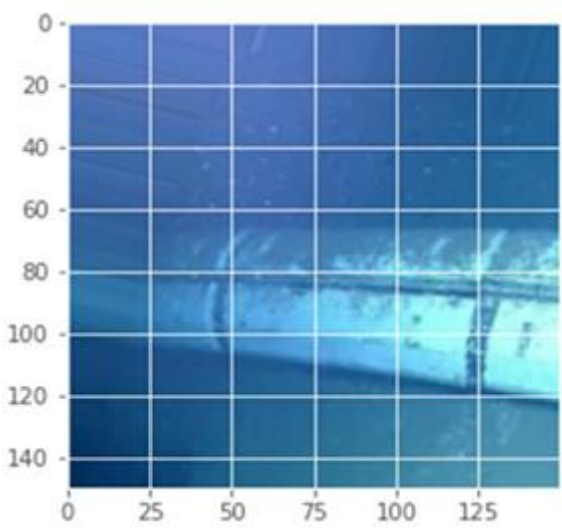

Fig. 6. Example of data augmentation

The hold-out validation method is chosen as the evaluation method, so the existing data is divided into three groups as training, testing, and validation datasets. Inputs and outputs are kept in the training dataset and created as part of the development of the prediction algorithm. The validation dataset is built to evaluate the model performance generated during the training phase of the network. The test dataset is the part where the neural network is tested and its performance is evaluated on new data that the network has not seen before. The network performance and overfitting status are evaluated by analyzing the performance of the test dataset.

The metrics to measure performance are determined by creating a confusion matrix, as it allows us to observe and control the system. The toggle event (yes or no) complexity for the matrix is derived from true positive, true negative, false positive, false negative values by examining the prediction question (is there any damage on the pipe). As a result, the certainty of prediction, precision, and F1-score values are calculated. Table 1 shows these values.

Table 1. Performance parameter values

\begin{tabular}{cc}
\hline Measure & Value \\
\hline Precision & 0.988 \\
Sensitivity & 0.975 \\
F1-Score & 0.981 \\
\hline
\end{tabular}

Filters are added to the images and the effect of mislabeled images on the loss function is minimized by adding noise parameters to the hidden layers and weights of the model. Feature extraction is performed through the convolution filter on the first layers using the convolutional neural network, and the increasingly abstracted features are placed on the feature map by transferring outputs to the subsequent layers. Edge detection filters are used to make clear sharp-soft and light-dark transitions on images, and padding is used to equalize the input-output size. Then pooling is done to reduce the size of the feature map and speed up the training of the network. 
Instead of processing all the data at the same time, the inputs can be divided into pieces by mini-batch size method and the data is processed in groups, thus achieving an economical solution in terms of time and memory.

RMSProp is selected as the optimization algorithm. RMSProp has its adaptive learning rate and is separate for each parameter.

ReLu activation function is used to normalize the output by using backward differentiation formula on hidden layers. ReLu activation function has been tested to work much faster than sigmoid and PreLu functions.

In order to prevent overfitting, regularization processes are implemented which reduced model complexity. By using the dropout layer method, approximately 0.5 dropout value is determined by drop outing on the hidden layers, and performance is increased by knot combinations and overfitting is prevented. Using the early stopping method, training is stopped and returned to the previous stage when the difference between validation and test losses begin to increase.

\section{Algorithm Performance of Underwater Pipeline Detection}

After the current data is analyzed and trained in the convolutional neural network, performance and losses are obtained for the training and validation seen in the graphics below. When the training and validation performance graph in Fig. 7 shows that training performance reaches $100 \%$ and validation performance is fixed at $75 \%$ on average.

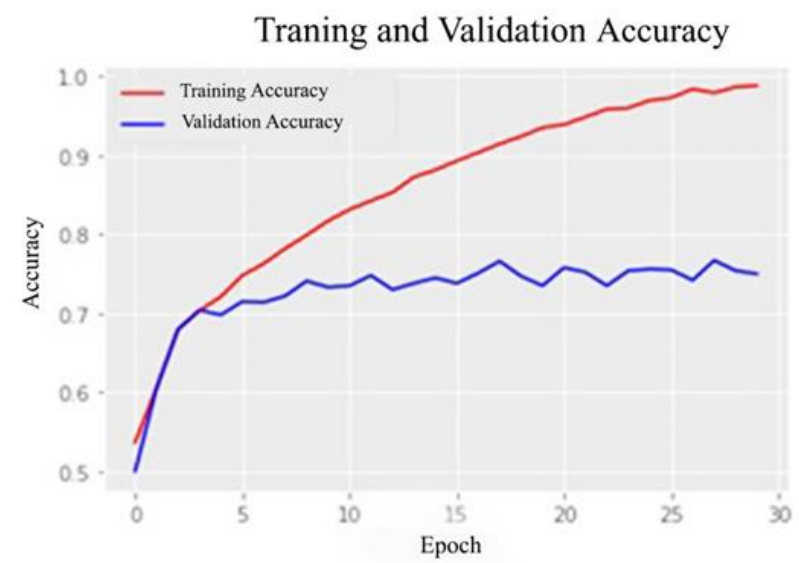

Fig. 7. Training and validation accuracy w. r. t. epoch

When the training and validation loss graph in Fig. 8 is examined, the training losses quickly approach zero, which is the 23 rd epoch of the validation. At this point, the epoch reaches its smallest value, and then the loss increases rapidly. This denotes that the model is overfitting.

In order to prevent overfitting of the model, the size of the dataset is increased by synthetic data generation, noise adding, and image generation and the network is regularized by the dropout layer and early stopping methods. To increase the speed of training, the number of layers is increased.

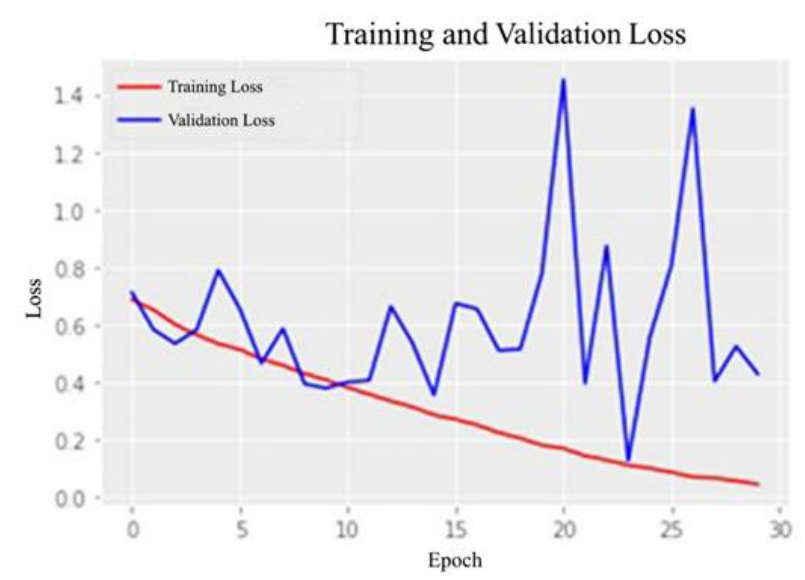

Fig. 8. Training and validation loss w. r. t. epoch

The training and validation performance graph in Fig. 9 shows that the training performance decreases and that the difference between training and validation decreases considerably.

When the loss graph in Fig. 10 shows that the training and validation losses are regularized. The performance rate of the test dataset is $83.02 \%$.

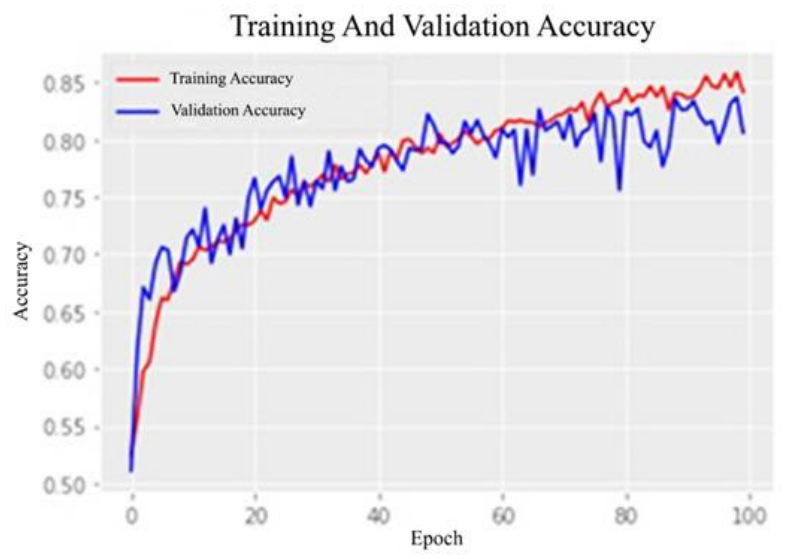

Fig. 9. Training and validation accuracy w. r. t. epoch Training And Validation Loss

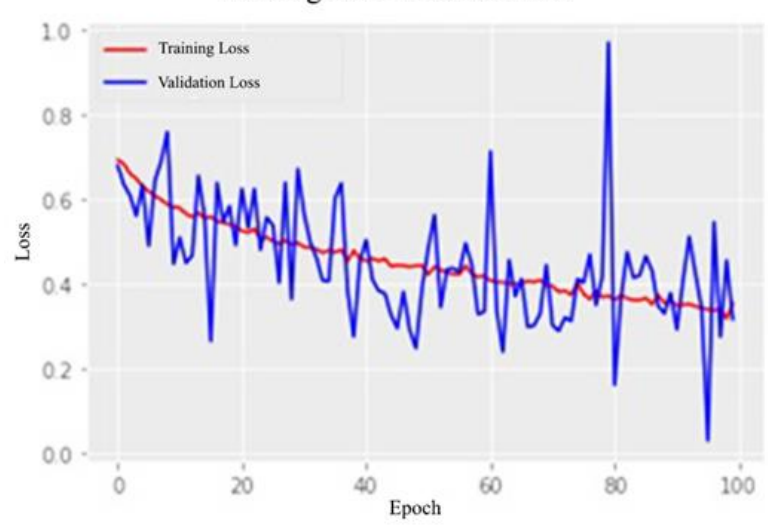

Fig. 10. Training and validation loss w. r. t. epoch 
The following performance and loss graphs are obtained for training and validation performance analysis when the noise of the curves in the above graphs is eliminated by the exponential correction method. The exponential correction method is a method of estimating the most recent past data from their exponentially greater weight. The performance rate on the test dataset increases greatly, reaching $97.63 \%$. Fig. 11 and Fig. 12 shows these results.

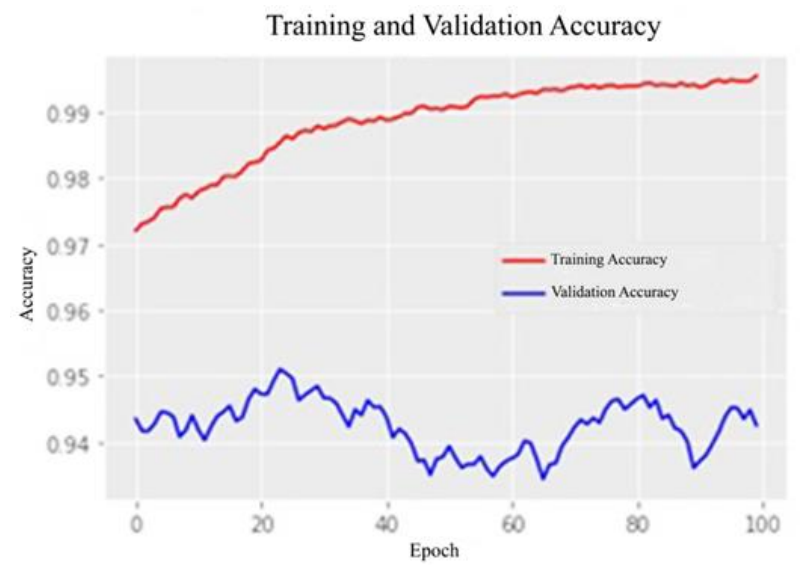

Fig. 11. Training and validation accuracy w. r. t. epoch

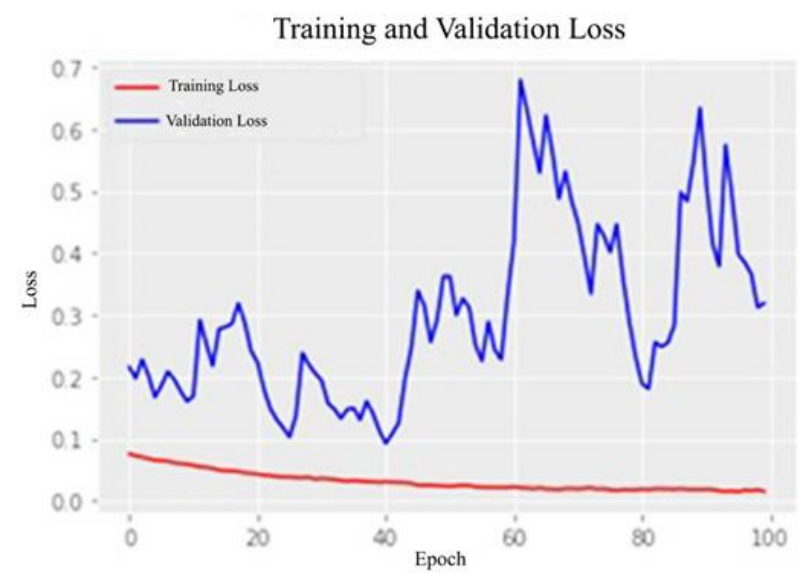

Fig. 12. Training and validation loss w. r. t. epoch

\section{Conclusions}

The use of image processing applications and artificial neural network techniques in order to detect leakages in an underwater oil and natural gas pipelines are preferred for problem-solving. Factors such as population growth and global growth are increasing the requirement and demand for oil and natural gas. Reliable, fast, and efficient transmission of energy is important both based on producer and consumer. This study provides a solution to the problem of leakage in an underwater oil and natural gas pipelines with an unmanned platform and provides a safe environment for underwater exploration in terms that do not put human lives at risk. Fast and high accuracy performance on images is achieved with the deep learning algorithm used in problem- solving. The problem is solved using convolutional neural networks with a performance rate of $97.63 \%$.

The study conducted with convolutional neural networks found that the quality of the dataset, the size of the dataset, the topology of the network, the number of layers, the dropout rate, and the depth of the model affect the performance and speed of the generated neural network significantly.

When the number of layers is increased and the depth of the artificial neural network is increased, feature map size is increased, and training results are better and training speed is decreased. It is observed that the mini-batch size value decreases the training speed when selected too small and the performance of the model when selected too large. Among the models created using different optimization and regularization methods and activation functions, the RMSProp optimization method, dropout layer, and early stopping regularization methods and ReLu activation function performed best. Hyperparameter optimization is found to be a requirement for deep learning applications, preventing overfitting, and reducing loss function value. The underwater pipeline will be detected by the CNN algorithm using our unmanned underwater vehicle at the future work. It has three thrusters shown as in Fig. 13. This vehicle is a remotely operated unmanned underwater vehicle that achieves the forward and depth motion and the rotation in the z-axis by thrusters. While the vehicle will be moving, the underwater video and images are captured with the camera located on the waterproof part of the vehicle. The online or offline underwater pipeline detection will be performed using these images that come from the vehicle.

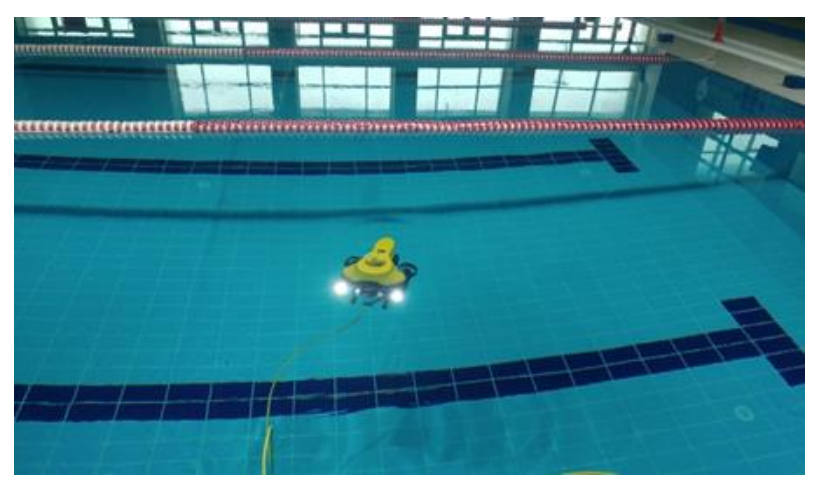

Fig. 13. Remotely operated unmanned underwater vehicle will be used future experimental study in the Blacksea

\section{ORCID}
A. Avc1
$0000-0002-9051-3816$
S. Karadeniz Kartal
0000-0003-4756-5490 


\section{References}

[1] S. Amidi, "https://stanford.edu/ shervine/l/tr/teaching/cs230/cheatsheet-convolutional-neural-networks," [Online].

[2] C. Roemer, "https://cp4space.wordpress.com/page/2/," [Online].

[3] F. Chollet, Deep Learning with Python, Manning, 2017.

[4] H. A. Song and Y. Lee, "Hierarchical Representation Using NMF," International Conference on Neural Information Processing, pp. 466-473, 2013.

[5] A. Gülcü and Z. Kuş, "Konvolüsyonel Sinir Ağlarında Hiper-Parametre Optimizasyonu Yöntemlerinin İncelenmesi," Gazi Üniversitesi Fen Bilimleri Dergisi , pp. 503-522, 2019.

[6] İ. Kurtoğlu, G. A. Canlı, M. O. Canlı and Ö. S. Tuna, "Dünyada ve Ülkemizde İnsansı Sualtı Araçları(ISAA-AUV\&ROV) Tasarım ve Uygulamalar1," GIDB|DERGİ, vol. 4, pp. 43-75, 2015.

[7] M. Dongfeng, C. Gui, Y. Lei and L. Zhigang, "Deepwater Pipeline Damage and Research on Countermeasure," Aquatic Procedia, pp. 180-190, 2015.

[8] K. A. Uysal and N. Cansever, "Doğalgaz ve Petrol Boru Hatlarında Hidrojenin Neden Olduğu Çatlamalar," in 3rd International Non-Destructive Testing Symposium and Exhibition, İstanbul, 2008.

[9] M. Graf, A. Liessem and K. R. Pöpperling, Review of the HIC Test Requirements for Linepipe over the years 1975 to 2000, Germany: Europipe, 1999.

[10] A. Jernelöv, "The Threats from Oil Spills: Now, Then, and in the Future," AMBIO, no. 39, pp. 353-366, 2010. 